\title{
Nerve growth factor and substance $P$ may be involved in moist exposed burn ointment-mediated chronic refractory wound healing
}

\author{
HUANG-DE FU ${ }^{1,2}$, SHU WANG $^{3}$, BIN GE $^{3}$, LI-QING LI ${ }^{3,4}$, \\ HONG-MENG ZENG ${ }^{3}$, QING-FENG SHU ${ }^{3}$ and YANG ZHOU ${ }^{3}$ \\ ${ }^{1}$ The First Affiliated Hospital of Jinan University, Guangzhou, Guangdong 510632; \\ ${ }^{2}$ Department of Neurosurgery, Affiliated Hospital of Youjiang Medical University for Nationalities; \\ ${ }^{3}$ Graduate School of Youjiang Medical University for Nationalities, Baise, Guangxi 533000; \\ ${ }^{4}$ Graduate School, Hunan University of Chinese Medicine, Changsha, Hunan 410208, P.R. China
}

Received September 25, 2017; Accepted June 21, 2018

DOI: $10.3892 /$ etm.2018.6390

\begin{abstract}
Moist exposed burn ointment (MEBO) is becoming increasingly popular in China as it shortens wound-healing time and reduces scar formation. However, its exact mechanism in mediating the wound-healing process is not yet clear. In the present study a total of 90 healthy adult male Wistar rats of specific-pathogen-free grade were divided equally into a control group, wound group, MEBO group, recombinant bovine basic fibroblast growth factor (rb-bFGF) group and sham operation group. Wound healing was observed from the extracted granulation tissues and recorded at three time points on 3, 7 and 14 days. Different levels of tumor necrosis factor $\alpha$ (TNF- $\alpha$ ) and interleukin-6 (IL-6) in tissue homogenate were detected using ELISA. Western blot analysis and quantitative PCR were used to detect the expression of nerve growth factor (NGF), substance $\mathrm{P}(\mathrm{SP})$ as well as tyrosine kinase A (TrkA) receptor protein and the corresponding mRNA levels in granulation tissue. It was observed that the wound healing progressed faster in the MEBO and rb-bFGF groups compared
\end{abstract}

Correspondence to: Dr Huang-De Fu, The First Affiliated Hospital of Jinan University, 601 West Huangpu Avenue, Guangzhou, Guangdong 510632, P.R. China

E-mail: bsfuhuangde@126.com

Abbreviations: ELISA, enzyme-linked immunosorbent assay; IL-6, interleukin-6; LSD, least square difference; MEBO, moist exposed burn ointment; NGF, nerve growth factor; NK1, neurokinin 1; PVDF, polyvinylidene difluoride; rb-bFGF, recombinant bovine basic fibroblast growth factor; SDS-PAGE, sodium dodecyl sulphate-polyacrylamide gel electrophoresis; SP, substance P; TCM, traditional Chinese medicine; TNF- $\alpha$, tumor necrosis factor $\alpha$; TrkA, tyrosine kinase A

Key words: nerve repair, moist exposed burn ointment, wound healing, nerve growth factor, tyrosine kinase A receptor, substance $\mathrm{P}$ with the wound group $(\mathrm{P}<0.01)$. TNF- $\alpha$ and IL-6 had an upward-downward trend at three time points, with the wound group demonstrating the most obvious increase $(\mathrm{P}<0.01)$. NGF and SP mRNA and protein levels in granulation tissue in MEBO, rb-bFGF and sham operation groups reached their highest levels on day 7 and then decreased on day 14. The expression level of TrkA was also measured simultaneously and its expression pattern was similar to that of NGF and SP. These results suggested that MEBO may promote nerve repair and accelerate wound healing through mediating the expression levels of NGF and SP, as well as TrkA.

\section{Introduction}

Skin is an extremely sensitive nerve-dependent tissue, which is recently considered as the largest organ of the body. Therefore, surface wound injury is often accompanied by nerve damage, and nerve repair ensues through the whole process of wound healing (1). Peripheral nerve damage in wounds can affect the wound-healing rate, and may cause paresthesia, affecting the patients' life quality, especially in case of chronic refractory healing wounds. Nerve regeneration is limited by damage, and if the duration of damage is more than 4 weeks, this may lead to the loss of functional recovery and regeneration (2).

Skin regeneration medical technology (namely moist medical technology) encompasses moist exposed burn ointment (MEBO) which is a new technique practiced in regenerative medicine (3). In recent years, this technology has been widely used in the treatment of chronic healing wounds, as an integral part of traditional Chinese medicine (TCM), and its efficacy has been recognized by many clinicians and medical professionals (4). After many years of improvement and development, MEBO has become a routine technical method that is widely used in many clinics. MEBO is mainly used to treat burns and a variety of body surface wounds with good results $(5,6)$, and is a currently recognized technique in the academic community (7-9). However, the molecular mechanisms of MEBO in the process of burn treatment have not been studied. It is currently understood that this technology 
launches four major biological reactions of hydrolysis, enzymatic hydrolysis, saponification and esterification via MEBO to keep the wound moist and create a suitable physiological environment for the healing process. While retaining healthy and repairing tissues, it liquefies necrotic tissue and discharges it layer by layer. This removes the necrotic barrier to healing and gives situ epidermal stem cells access to the wound bed to allow physiological regeneration and wound repair (10). At the same time, MEBO promotes activation and proliferation of epidermal stem cells and enhances the expression of the epidermal stem cells marker CK19 (11).

Many factors are involved in the natural wound healing process. Nerve growth factor (NGF), the first member of the family of neurotrophic factor discovered, can promote the development and differentiation of the central and peripheral nervous system, and is identified as one of the important factors in nerve injury repair. It plays a key role in axonal growth, cell differentiation, cell migration, promotion and control of nerve regeneration $(12,13)$. NGF acts as a signaling molecule by binding two types of trans-membrane protein receptors, namely the neurotrophic factor receptor (p75NTR) and the tyrosine kinase A (TrkA) receptor $(14,15)$. These trans-membrane proteins exhibit different binding efficiency, showing low affinity by the p75NTR and a higher affinity by TrkA (16). NGF combines with TrkA receptor to play a positive regulation by activating a series of phosphorylation cascades that promote neuronal growth, survival and differentiation (17). NGF binds with P75NTR to promote endothelial cell apoptosis. It was showed that the growth rate and quality of axons can be improved by these neurotrophic factors (18). Therefore, we hypothesized that NGF may be involved in MEBO burn therapy.

Substance P (SP) is a common neuropeptide composed of 11 amino acids that belongs to the tachykinin family. As a neurotransmitter or neuromodulator, it plays an important role in the nervous system. Neurokinin 1 (NK1) receptor is the specific receptor for SP $(19,20)$. Exogenous SP can stimulate the proliferation and differentiation of fibroblasts on the wound surface, accelerate synthesis and secretion of angiogenesis factors and collagen fibers, and play a significant role in wound healing and scar hyperplasia (21). On damage skin, the sensory nerve endings release SP causing a long-term neuroendocrine response aiding the cell repair, which may be the reason for the high levels of SP found at injury sites. In scar tissue, fibroblasts simultaneously up-regulate fibroblast growth factors and receptors in the granulation tissue, further amplifying the reparative effects of SP. A previous study revealed that the SP released by nerve endings could induce and elevate the levels of SP released from scar fibroblasts (22). Therefore, we hypothesized that SP may be associated with the burn recovery process of MEBO.

This study was aimed to investigate the effects of MEBO on the expression of NGF, TrkA and SP in chronic refractory granulation tissue of rats, and to unravel the possible mechanisms of MEBO in the promotion of nerve regeneration and repair in chronic refractory wounds.

\section{Materials and methods}

Reagents. MEBO was obtained from Shantou Special Economic Zone MEBO Pharmaceutical Co., Ltd. (Shantou,
China), Beijing Guangming Chinese Medicine Burns and Urology Institutem [Beijing, China; State Food and Drug Administration (SFDA), approval no. Z20000004]. Recombinant bovine basic fibroblast growth factor (rb-bFGF) was obtained from Essex Bio-Technology Co., Ltd. (Zhuhai, China; SFDA, approval no. S19991021). Chloral hydrate was obtained from Haoshen Chemical Trading Co., Ltd. [Taixing, China), Chemical Abstracts Service (CAS), no. 302-17-0]; the Hydrocortisone acetate injection was obtained from Shanghai General Pharmaceutical Co., Ltd., (Shanghai, China; SFDA, approval no. H31021290). The Rat TNF- $\alpha$ ELISA kit and Rat IL-6 ELISA kit were from RapidBio (West Hills, CA, USA); the anti-NGF antibody and anti-TrkA antibody were from Abcam (Cambridge, UK); the anti-SP antibody was from Santa Cruz Biotechnology, Inc. (Dallas, TX, USA); the horseradish peroxidase labeled goat anti-rabbit antibody and $\beta$-actin antibody were from Zhongshan Jinqiao Biotechnology, (Beijing, China); the Real-Time quantitative PCR kit, reverse transcription kit and RNA extraction kit were from Takara Bio, Inc. (Otsu, Japan).

Animals. Adult Wistar male rats of specific-pathogen-free (SPF) grade (12 weeks old, weighing between 220-250 g) were provided by the Youjiang National Medical College Animal Experimental Center. The rats were kept in the SPF grade laboratory of the animal experiment center, Youjiang National Medical College with controlled humidity of $55-75 \%$, and an indoor temperature of $20-25^{\circ} \mathrm{C}$ with good ventilation. After 1-week acclimatization, the rats were used for generating the experimental models, which was approved by the Ethics Committee of the Youjiang National Medical College and adhered to the National Laboratory for Experimental Animal Care published by the National Institutes of Health (NIH publication no. 85-23, 1996).

Chronic refractory wound model. The animal models created in this study adopted the whole layer skin defection method established by Zhao et al (23) and the quantitative method of plastic ring granulomamodified by Shen (24). We further improved these methods and created the rat model of chronic refractory wounds. Rats were anesthetized with intraperitoneal injection of $7 \%$ chloral hydrate at $280 \mathrm{mg} / \mathrm{kg}$. The rats' back hair was shaved with an electric razor, and then Nair hair removal cream was used to ensure complete hair removal. The rats were then washed with water and their back skin was dried and placed at $28-30^{\circ} \mathrm{C}$. When recovering naturally, the rats were given free access to diet for more than $24 \mathrm{~h}$ to eliminate the effect of hair removal agent on the skin damage. The rats were then anesthetized again in the same manner. We marked with a $1.5 \mathrm{~cm}$ diameter circular stamp to ensure a consistent model area. Under sterile conditions, two whole layer skin wounds were made along the direction of the spine using surgical methods, which were deep in the fascia and $1.5 \mathrm{~cm}$ in diameter. The two wounds were covered with two layers of dry gauze and fixed with tape in a '丰' shape. After modeling, hydrocortisone acetate was immediately injected intraperitoneally $(6 \mathrm{mg} / 100 \mathrm{~g}$ body weight). At this point, a chronic refractory wound model was established.

Experimental animal grouping and treatment. A total of 90 rats were divided into control group (18 rats), sham 
operation group (18 rats) and chronic refractory wound group (54 rats). The rats in the control group were treated without damage. The rats in the sham operation group were treated following the basis of the above model, where they underwent the whole layer skin resection at the model site, but without hydrocortisone injection. The rats in the chronic refractory wound group were randomly sub-divided into the wound group, MEBO group and rb-bFGF group $(n=18$ in each group). After the wounds were created, we immediately provided treatment. 1/5,000 nitrofurazone liquid was used to clean wounds before the first dressing, and after that saline was applied to clean the wound before each dressing. Each group underwent the corresponding dressing treatment twice daily (morning and evening).

In the MEBO group, wounds were covered with two layers of gauze that contained MEBO. The preparation of MEBO gauze $\left(0.2 \mathrm{~g} / \mathrm{cm}^{2}\right)$ was based on the Clinical Application Specification of Burned Skin Regenerative Medical Technology (25).rb-bFGF, as an exogenous growth factor was selected as the control drug, which can improve the wound's lack of endogenous growth factors and can induce vascular endothelial cells and fibroblast proliferation to promote wound healing. rb-bFGF is now widely used in the treatment of trauma, burns and other wounds (26). In the rb-bFGF group, the wound surface was covered with two layers of gauze $\left(60 \mathrm{U} / \mathrm{cm}^{2}\right)$ impregnated with rb-bFGF. In the wound group and sham operation group, wound surfaces were covered with two layers of gauze that contained saline. In the control group, the skin was covered over the corresponding site with two layers of saline gauze. The gauze was cut into small pieces according to the size of the wound and stuck to the wound, and then covered with another two layers of dry gauze and fixed with tape.

Specimen preparation. In a preliminary experiment, we found that the duration of wound healing in rats was approximately 14 days. Six rats were randomly selected from each group at three time points, namely 3, 7 and 14 days and were culled by cervical dislocation. The entire wound granulation tissue $(0.5 \mathrm{~cm}$ from the wound margin) was collected under the wound deep fascia from both sides of the spine. The tissues were then carefully cut into small pieces, rinsed with saline, and placed in a cryopreservation tube then transferred to a $-80^{\circ} \mathrm{C}$ freezer for storage in a liquid nitrogen tank.

Wound healing rate. Wound healing in all 5 groups was observed. The wound area was measured and recorded daily to calculate the wound-healing rate. Wound healing rate $=$ (area before administration-non-healing wound area)/area before administration $\mathrm{x} 100 \%$.

ELISA test. The levels of tumor necrosis factor $\alpha(\mathrm{TNF}-\alpha)$ and interleukin-6 (IL-6) were measured by enzyme-linked immunosorbent assay (ELISA), and the procedure was carried out according to the manufacturer's instructions.

Quantitative PCR experiment. RNA extraction and cDNA preparation were performed according to the experimental method provided by the TAKARA kit. The extracted cDNA was used as template to conduct amplification and expression determination according to the kit instructions. Primers were designed and synthesized by Takara Biotechnology Co., Ltd., Dalian, China (Table I). The amplification expression rate of detected $\mathrm{Cq}$ values was calculated by $2^{-\Delta \Delta \mathrm{Cq}}$ method.

Western blotting. The tissues were homogenized by grinding manually in liquid nitrogen. After protein extraction, protein concentrations were determined and then adjusted to equal amount of protein levels for all samples before protein denaturation and storage in at $-20^{\circ} \mathrm{C}$ freezer. Protein concentrations were assessed using the Bradford method. $30 \mu \mathrm{g}$ protein was separated by $12 \%$ sodium dodecyl sulphate-polyacrylamide gel electrophoresis (SDS-PAGE), and then transferred to polyvinylidene difluoride (PVDF) membrane followed by blocking the PVDF membrane with 5\% skim milk for $1 \mathrm{~h}$. The PVDF membranes were then incubated with the anti-NGF antibody $(1: 1,000)$, anti-SP antibody $(1: 1,000)$ and anti-TrkA antibody $(1: 1,000)$, respectively overnight at $4^{\circ} \mathrm{C}$. The membranes were washed with TBST for 4 times ( 5 min each time). Subsequently the membranes were incubated with an appropriate secondary antibody for $1 \mathrm{~h}$ at room temperature. After washing with TBST, the membranes were exposed using chemiluminescence system and the gel image system was used to analyze the gray value of target protein. $\beta$-actin $(1: 1,000)$ was selected as internal reference. The protein content of the sample was expressed as the ratio of the absorbance of the target band to the internal control band.

Statistical analysis. SPSS 20.0 software (IBM Corp., Armonk, NY, USA) was used for statistical analysis. The data were expressed as mean \pm standard deviation and the means of two groups were compared by t test. The comparison between the means of three or more independent groups was based on one-way analysis of variance (ANOVA) and comparison between two means among all the groups was done by Fisher's least significant difference (LSD) test. $\mathrm{P}<0.05$ was considered to indicate a statistically significant difference.

\section{Results}

Rats in MEBO and rb-bFGF treatment groups showed a higher healing rate. All experimental groups showed wound healing over time. The wound healing rate in each group was as follows: Control group $>$ sham operation group $>\mathrm{MEBO}$ group $=$ rb-bFGF group $>$ wound group (Fig. 1). The results showed that the rats in MEBO and rb-bFGF groups could benefit by significantly increased wound healing from MEBO and $\mathrm{rb}-\mathrm{bFGF}$ treatment compared to the wound group $(\mathrm{P}<0.01)$.

$M E B O$ and $r b-b F G F$ treatment significantly down-regulated the expression of $T N F-\alpha$ and $I L-6$. Compared with the control group, the levels of TNF- $\alpha$ and IL- 6 in wound tissue homogenate decreased with time in the wound group, MEBO group, rb-bFGF group and sham operation group. The levels of TNF- $\alpha$ and IL- 6 in the sham operation group showed the highest significant decrease $(\mathrm{P}<0.01)$ while those in the wound group showed the lowest decrease $(\mathrm{P}<0.01)$. The levels of TNF- $\alpha$ and IL-6 in rats within each group were as follows: Wound group $>$ MEBO group=rb-bFGF group $>$ sham operation group $>$ control group (Fig. 2), and the difference was statistically significant $(\mathrm{P}<0.01)$. The results showed that 
Table I. Sequences of primers used for quantitative PCR.

\begin{tabular}{lllr}
\hline Name of the gene & \multicolumn{1}{c}{ Forward Primer sequence } & \multicolumn{1}{c}{ Reverse Primer sequence } & Size (bp) \\
\hline GAPDH & 5'-GGCACAGTCAAGGCTGAGAATG-3' & 5'-ATGGTGGTGAAGACGCCAGTA-3' & 143 \\
SP & 5'-CGCCGATGTTTCAGTCCATTC-3' & 5'-GACGTATTCAGTCCGTGTTGGTTG-3' & 96 \\
TrkA & 5'-TGCTCAACAAATGTGGACAGAGG-3' & 5'-TGTCATGAAGTGTAGGGACATGG-3' & 98 \\
NGF & 5'-TGCCAAGGACGCAGCTTTC-3' & 5'-TGAAGTTTAGTCCAGTGG GCTTCAG-3' & 171 \\
\hline
\end{tabular}

SP, substance P; TrkA, tyrosine kinase A; NGF, nerve growth factor.

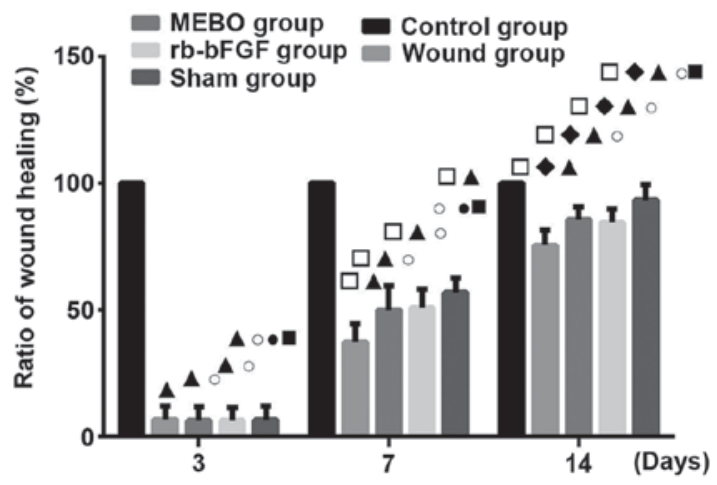

Figure 1. Ratio of wound healing at three time points. ${ }^{\mathrm{P}} \mathrm{P}<0.01$ compared with 3 days; ${ }^{\bullet} \mathrm{P}<0.01$ compared with 7 days; ${ }^{\wedge} \mathrm{P}<0.01$ compared with the Control group; ${ }^{\circ} \mathrm{P}<0.01$ compared with the Wound group; ${ }^{\bullet} \mathrm{P}<0.01$ compared with the MEBO group; $\mathrm{P}<0.01$ compared with the rb-bFGF group. MEBO, moist exposed burn ointment; rb-bFGF, recombinant bovine basic fibroblast growth factor.

MEBO and rb-bFGF treatment in MEBO and rb-bFGF groups could significantly decrease the expression of TNF- $\alpha$ and IL-6 compared with the wound group $(\mathrm{P}<0.01)$.

Comparison of NGF mRNA transcription and protein expression. Compared with the control group, the protein expression and mRNA transcription level of NGF in rats wound tissue of MEBO group, rb-bFGF group and sham operation group showed an upward-downward trend at 3 time points, and reached its highest level at day 7 and decreased at day 14 (Figs. 3 and 4). However, rats in the wound group showed no decrease in NGF mRNA on day 14 $(\mathrm{P}<0.01$; Figs. 3 and 4$)$. The rats in control group displayed constant levels of NGF mRNA and protein $(\mathrm{P}>0.05$; Figs. 3 and 4). On day 3, the expression of NGF mRNA in the wound tissue of the 5 groups was as follows: Sham operation group $>$ rb-bFGF group $=$ MEBO group $>$ control group $>$ wound group (Fig. 4A), while difference in the expression level of NGF protein was not statistically significant between groups ( $\mathrm{P}>0.05$; Figs. 3 and 4B). On day 7, the expression of NGF mRNA in wound tissue of the 5 groups was as follows: Sham operation group $>$ rb-bFGF group $=$ MEBO group $>$ wound group $>$ control group (Fig. 4A), and the expression of NGF protein was consistent with the change in mRNA (Figs. 3 and 4B). On day 14, the expression of NGF mRNA in wound tissue of 5 groups were as follows: Wound group $>$ rb $-b F G F$ group $=$ MEBO group $=$ sham operation group $=$ control group (Fig. 4A) and the expression of NGF protein was consistent with the changes of mRNA (Figs. 3 and 4B). The above results suggested that NGF was directly involved in the MEBO and rb-bFGF-mediated wound repair processes.

Comparison of TrkA mRNA transcription and protein expression. TrkA expression was examined in all groups on days 3 , 7 and 14 (Figs. 3 and 5). Compared with the control group, the protein expression and mRNA transcription level of TrkA in wound tissue of the MEBO group, rb-bFGF group and sham operation group had an upward-downward trend at three time points, reaching their highest levels on day 7 and decreasing on day 14 (Figs. 3, and 5CA and B). The levels of TrkA in the wound group showed a continually increasing trend with the highest levels on day 14 . The difference among groups was statistically significant $(\mathrm{P}<0.01$; Fig. $5 \mathrm{~A}$ and $\mathrm{B})$. The rats in the control group showed no significant difference in TrkA protein and mRNA transcription expression levels at the 3 time points ( $\mathrm{P}>0.05$; Figs. 3 and $5 \mathrm{~A})$. On day 3 , the expression of TrkA protein in the wound tissue of the 5 groups were as follows: Sham operation group $>$ rb-bFGF group=MEBO group $>$ control group $>$ wound group (Fig. 5A), and the expression of TrkA protein was consistent with the changes in mRNA levels (Figs. 3 and 5B). On day 7, the expression of TrkA protein in the wound tissue of the 5 groups was as follows: Sham operation group $>$ rb-bFGF group $=$ MEBO group $>$ wound group $>$ blank group (Figs. 3 and 5A), and the protein expression of TrkA was consistent with the changes in mRNA levels (Figs. 3 and 5B). On day 14, the protein expression of TrkA in the wound tissue of the 5 groups was as follows: Wound group $>$ rb-bFGF group=MEBO group=sham operation group=blank group (Figs. 3 and 5A), and the expression of TrkA protein was consistent with the changes in mRNA levels (Figs. 3 and 5B). The results suggested that TrkA was involved in MEBO and rb-bFGF-mediated wound repair processes.

Comparison of mRNA transcription and protein expression of SP. SP expression was examined in all groups on days 3, 7 and 14 (Figs. 3 and 6). Compared with control group, the protein expression and mRNA transcription level of SP in the MEBO group, rb-bFGF group and sham operation group presented an upward-downward trend at 3 time points, and reached the peak level at 7 days and decreased on the 14th day $(\mathrm{P}<0.01$; Figs. 3, and $6 \mathrm{~A}$ and $\mathrm{B})$. The mRNA levels in the wound group showed an upward trend with the highest expression level on day 14 . The protein levels showed only a slight decrease, and was much less than the decrease in 

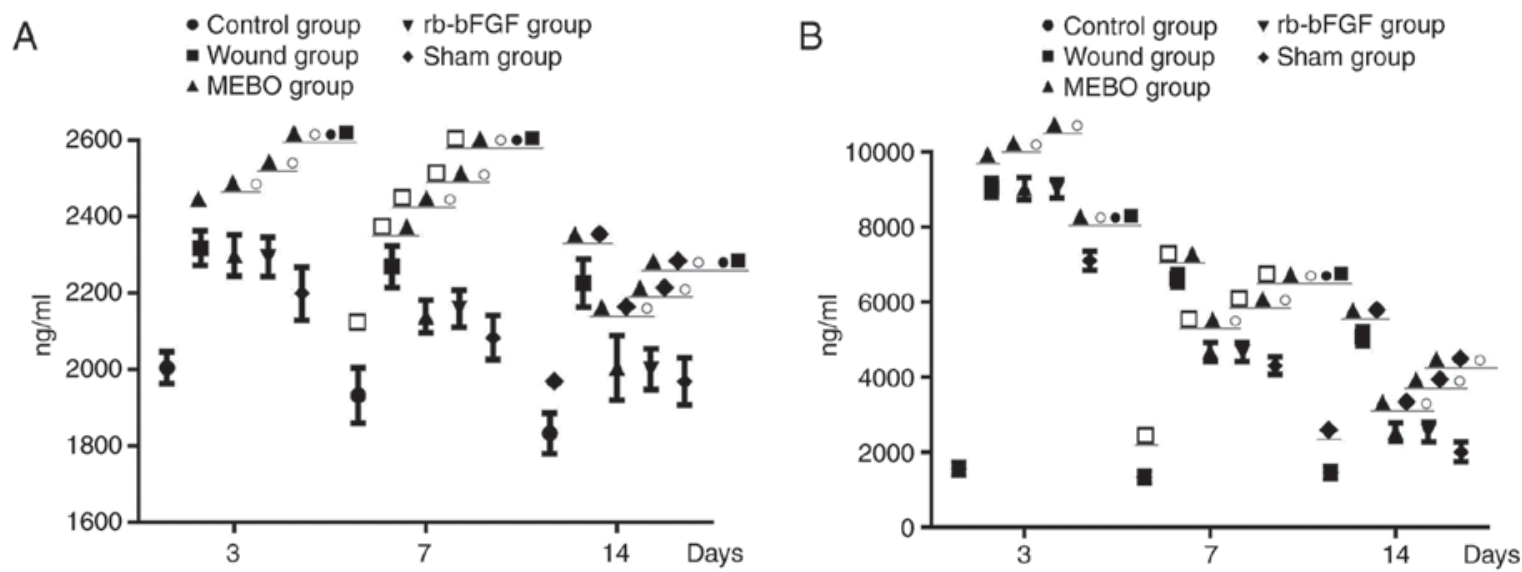

Figure 2. The expression of inflammatory factors. Comparison of inflammatory factor expression levels of TNF- $\alpha$ (A) and IL-6 (B) in the granulation tissue of rats at three time points. ${ }^{\square} \mathrm{P}<0.01$ Compared with 3 days; ${ }^{\circ} \mathrm{P}<0.01$ compared with 7 days; ${ }^{\wedge} \mathrm{P}<0.01$ compared with the Control group; ${ }^{\circ} \mathrm{P}<0.01$, compared with the Wound group; ${ }^{\bullet} \mathrm{P}<0.01$ compared with the MEBO group; ${ }^{-\mathrm{P}}<0.01$ compared with the rb-bFGF group. TNF- $\alpha$, tumor necrosis factor $\alpha$; IL-6, interleukin- 6 ; rb-bFGF, recombinant bovine basic fibroblast growth factor; MEBO, moist exposed burn ointment.

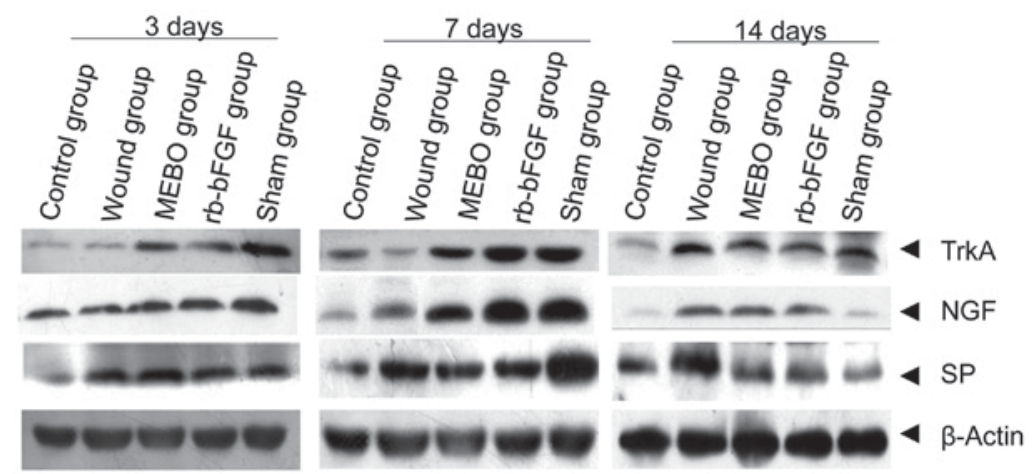

Figure 3. The expression of TrkA, NGF and SP at the protein level. Comparison of TrkA, NGF and SP proteins in the granulation tissue of rats at three time points was indicated. TrkA, tyrosine kinase A; NGF, nerve growth factor; SP, substance P; rb-bFGF, recombinant bovine basic fibroblast growth factor; MEBO, moist exposed burn ointment.
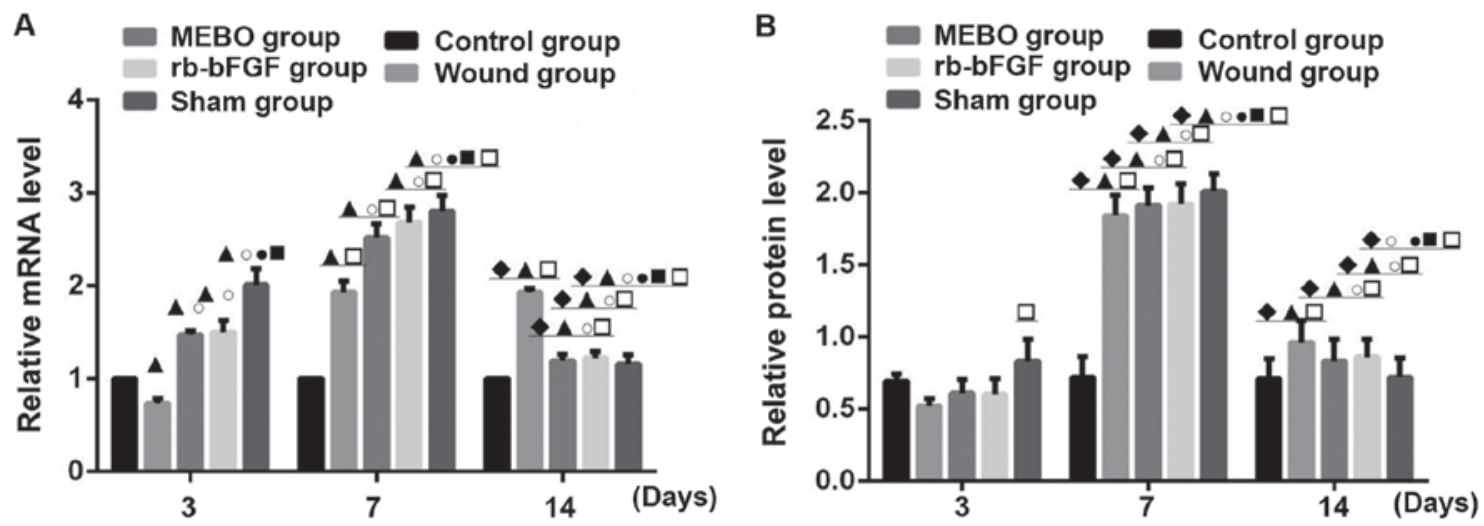

Figure 4. The expression of NGF at indicated points. Comparison of NGF (A) mRNA and (B) protein expression levels in the granulation tissue of rats at three time points. ${ }^{\square} \mathrm{P}<0.01$ Compared with 3 days; ${ }^{\circ} \mathrm{P}<0.01$ Compared with 7 days; ${ }^{\Delta} \mathrm{P}<0.01$ Compared with the Control group; ${ }^{\circ} \mathrm{P}<0.01$ Compared with the Wound group; $\bullet \mathrm{P}<0.05$ Compared with the MEBO group; $" \mathrm{P}<0.01$ Compared with the rb-bFGF group. NGF, nerve growth factor; MEBO, moist exposed burn ointment; rb-bFGF, recombinant bovine basic fibroblast growth factor.

the other experimental groups, and the difference between groups was statistically significant $(\mathrm{P}<0.01$; Figs. 3 and $6 \mathrm{~A})$. The rats in the control group showed no significant difference in SP protein and mRNA transcription expression levels between the 3 time points $(P>0.05$; Figs. 3 and $6 \mathrm{~A})$.
On day 3, the mRNA expression level of SP in the wound tissue of the 5 groups was as follows: Sham operation group $>$ rb-bFGF group=MEBO group $>$ control group $>$ wound group (Fig. 6A) and the expression of SP protein was consistent with the changes in mRNA levels (Fig. 6B). On day 7, 

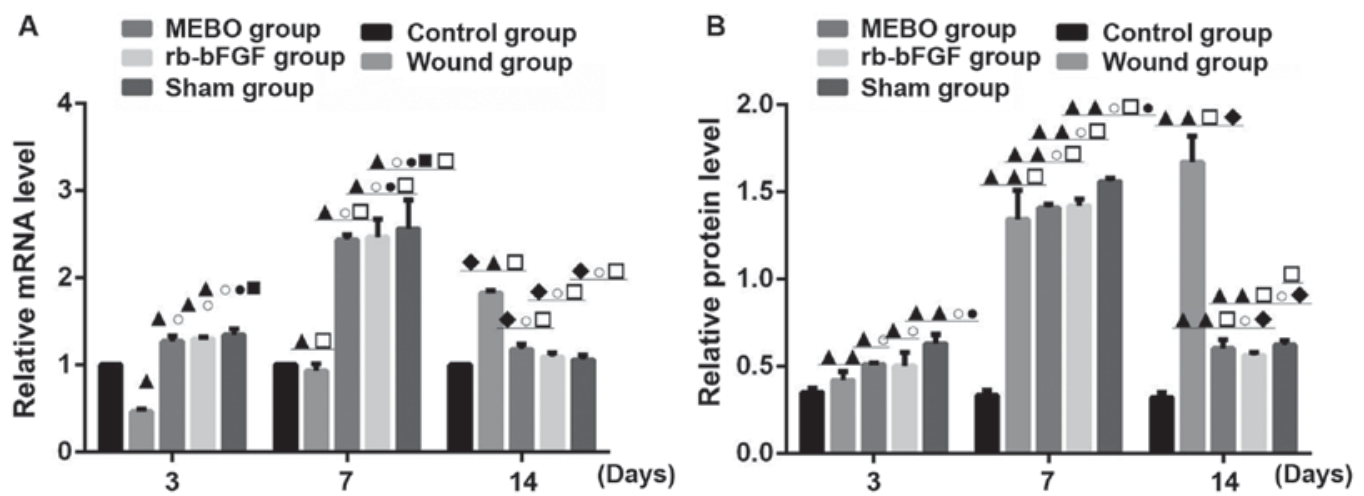

Figure 5. The expression of TrkA at indicated points. Comparison of TrkA mRNA (A) and protein (B) expression levels in the granulation tissue of rats at three time points. ${ }^{\square} \mathrm{P}<0.01$ Compared with 3 days; ${ }^{\mathrm{P}} \mathrm{P}<0.01$ Compared with 7 days; ${ }^{\mathbf{A}} \mathrm{P}<0.01$ Compared with the Control group; ${ }^{\circ} \mathrm{P}<0.01$ Compared with the Wound group; ${ }^{\mathrm{P}}<0.05$ Compared with the MEBO group; $\mathrm{P}<0.01$ Compared with the rb-bFGF group. TrkA, tyrosine kinase A; rb-bFGF, recombinant bovine basic fibroblast growth factor; MEBO, moist exposed burn ointment.
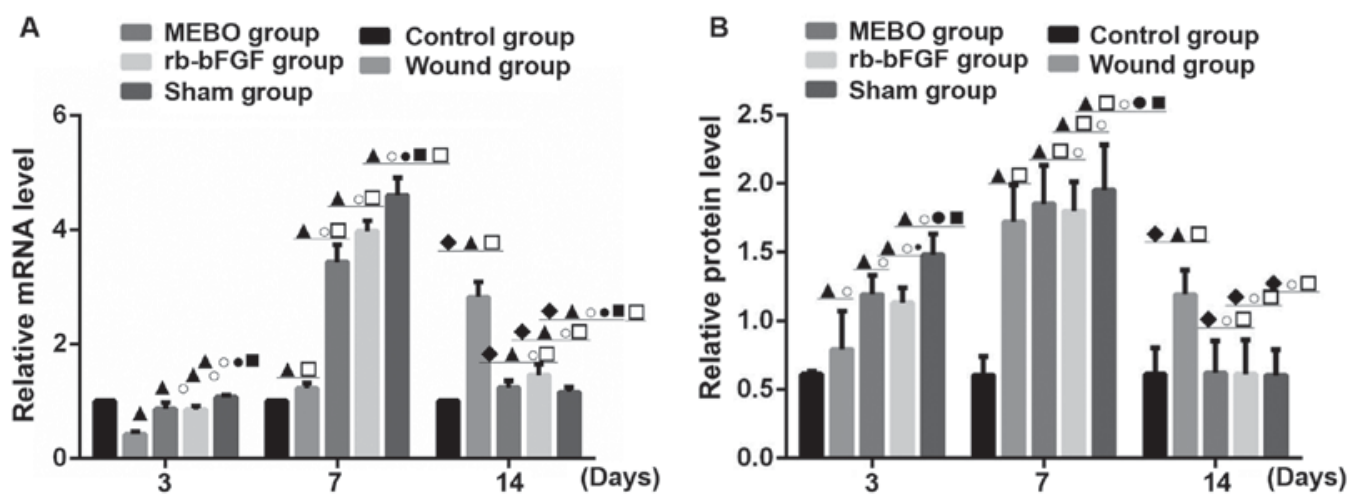

Figure 6. The expression of SP at indicated points. Comparison of SP mRNA (A) and protein (B) expression levels in the granulation tissue of rats at three time points. ${ }^{\square} \mathrm{P}<0.01$ Compared with 3 days; ${ }^{\bullet} \mathrm{P}<0.01$ Compared with 7 days; ${ }^{\wedge} \mathrm{P}<0.01$ Compared with the Control group; ${ }^{\circ} \mathrm{P}<0.01$ Compared with the Wound group; $\bullet P<0.05$ Compared with the MEBO group; " $\mathrm{P}<0.01$ Compared with the rb-bFGF group. SP, substance P; rb-bFGF, recombinant bovine basic fibroblast growth factor; MEBO, moist exposed burn ointment.

the mRNA expression levels of SP in the wound tissue of the 5 groups was as follows: Sham operation group $>$ rb-bFGF group=MEBO group $>$ trauma group $>$ control group (Fig. 6A) and the protein expression levels of SP was consistent with the changes in mRNA levels (Fig. 6B). On day 14, the mRNA expression of SP in the wound tissue of the 5 groups was as follows: Sham operation group $>$ rb-bFGF group=MEBO group $>$ wound group $>$ control group (Fig. 6A) and the expression of SP protein was consistent with the changes in mRNA levels (Fig. 6B). The results suggested that SP was involved in MEBO and rb-bFGF-mediated wound repair processes.

\section{Discussion}

The aim of this study was to investigate the effects of MEBO on the expression of NGF, TrkA and SP in chronic refractory granulation tissue of rats to unravel the possible mechanisms of therapeutic benefits associated with wound-healing. This is the first report to suggest that MEBO may promote wound healing by mediating NGF, TrkA, SP mRNA and protein expression, providing a novel insight into wound healing.

The initial inflammatory response is important for effective wound healing, but if the initial response is too high it can delay the healing process (27). The results of this study showed that intervention with either MEBO or rb-bFGF increased wound-healing rates and reduced TNF- $\alpha$ and IL-6 overexpression in wound homogenate tissue, especially on the 7th day during treatment, and MEBO showed a more obvious effect. The improvement in wound healing was consistent with similar previous studies conducted with MEBO (5-9). Because MEBO and rb-bFGF do not contain anti-inflammatory drugs, we speculated that MEBO indirectly reduces the expression of some inflammatory factors, during wound-healing and improves the wound's microenvironment, thereby creating a positive physiological environment for wound cells to speed up the wound healing process.

In order to understand the potential mechanism of MEBO, we investigated factors that have been shown to have important roles in wound healing (12-22). The results showed that the levels of NGF, TrkA, SP protein and mRNA in the wounds of rats had an upward trend until day 7 and then decreased again on day 14. Contrarily, in the control group, their levels remained constant and in the wound group it either continued to increase till day 14 , or did not decrease as rapidly as in the other experimental groups. So, these results suggest that the healing rate in chronic refractory wounds treated with either MEBO or rb-bFGF was close to that of the 
normal wound physiological healing level seen in the sham operation group. Rats in the wound group did not receive any drug intervention, so wound healing was slow, resulting in higher protein and mRNA levels of NGF, TrkA and SP at 3 time points.

However, this study has some limitations. Due to the complexity of the signaling pathways involved, this study has revealed only a part of the mechanism involved in MEBO promoting chronic refractory wound healing. With regard to the specific mechanism much remains to be investigated including the complete signaling pathways and the multi-channel interactions. In addition, we have evaluated the wound-healing process by collecting the parameters during three time points only, which may not fully explain the entire wound healing process. As MEBO is a TCM technique with complex interactions between many different factors, the details of the active factors involved in wound healing still need to be evaluated and investigated.

In conclusion, the results of this study showed that MEBO promoted wound repair and accelerated the wound healing in a similar way as application of rb-bFGF. The mechanism may involve the regulation of inflammatory response and the expression of NGF, TrkA and SP. However, more specific details of the mechanism remain to be investigated to understand the complete signaling pathways and multi-channel interactions involved.

\section{Acknowledgements}

This study was supported by a grant from the National Natural Science Foundation of China (grant no. 81560776).

\section{Competing interests}

The authors declare that they have no competing interests.

\section{References}

1. Huang Y, Qi SH, Shu B, Mao RX, Xie JL and Xu YB: In vivo study on the effect of Skin-derived precursors (SKPs) on neural repair in skin wounds. Chin J Nerv Mental Dis 37: 531-534, 2011 (In Chinese)

2. Sulaiman W and Dreesen TD: Effect of local application of transforming growth factor- $\beta$ at the nerve repair site following chronic axotomy and denervation on the expression of regeneration-associated genes. Laboratory investigation. J Neurosurg 121 859-874, 2014

3. Xu RQ: Theory and practice of regenerative medicine: A speech at the 7th National Academic conference on Burns and Wounds. Chin J Burns Wound Surf Ulcers 14: 201-215, 2002 (In Chinese).

4. Mabrouk A, Boughdadi NS, Helal HA, Zaki BM and Maher A: Moist occlusive dressing (Aquacel((®) Ag) versus moist open dressing $\left(\mathrm{MEBO}\left({ }^{\circledR}\right)\right)$ in the management of partial-thickness facial burns: A comparative study in Ain Shams University. Burns 38: 396-403, 2012.

5. Tang QL, Han SS, Feng J, Di JQ, Qin WX, Fu J and Jiang QY: Moist exposed burn ointment promotes cutaneous excisional wound healing in rats involving VEGF and bFGF. Mol Med Rep 9: 1277-1282, 2014.

6. Tang QL, Huang X and Wang Y: Ultrastructural Pathology and the Mechanism of Expression of MAPKK mRNA \& c-myc mRNA of Chronic Refractory Wound Treated by MEBT/MEBO. Chin Gen Pract 18: 294-299, 2015 (In Chinese).
7. Li NQ: New Century National College of Integrative Medicine clinical medicine professional planning teaching materials 'integrated traditional Chinese and Western medicine'. China Traditional Chinese Medicine Press, Bejing, 2005.

8. He QH: National traditional chinese medicine industry higher education '12th five-year' national planning textbook 'integrative medicinesurgery', 2nd edition. China Traditional Chinese Medicine Press, Bejing, 2014.

9. $\mathrm{He} \mathrm{QH}$ : National traditional chinese medicine industry higher education '13th five-year' national planning textbook 'integrative medicine surgery'. 3rd edition. China Traditional Chinese Medicine Press, Bejing, 2016.

10. Xu RQ and Xiao M: The mechanism of burn regenerative therapy and wound healing. Chin J Burns Wounds Surface Ulcers 15: 253-261, 2003 (In Chinese).

11. El-Hadidy MR,El-Hadidy AR, Bhaa A, Asker SA and Mazroa SA: Role of epidermal stem cells in repair of partial-thickness burn injury after using moist exposed burn ointment (MEBO(®)) histological and immunohistochemical study. Tissue Cell 46: 144-151, 2014.

12. Muir D: The potentiation of peripheral nerve sheaths in regeneration and repair. Exp Neurol 223: 102-111, 2010.

13. Griffin JW, Hogan MV, Chhabra AB and Deal DN: Peripheral nerve repair and reconstruction. J Bone Joint Surg Am 95: 2144-2151, 2013

14. Schecterson LC and Bothwell M: Neurotrophin receptors: Old friends with new partners. Dev Neurobiol 70: 332-338, 2010.

15. Xu YF: Effects of FGF19-FGFR4 and NGF-TrkA Signaling Pathways on Progression and Prognosis of Cholangiocarcinoma. PhD dissertation, Shandong University, 2014.

16. Yang XQ: The preliminary study of expression and interaction of EGFR family and NGF/TrkA in cholangiocarcinoma. PhD dissertation, Shandong University, 2014.

17. Ji YX: Expression of Nerve Growth Factor in Diabetic Foot Wound Tissue. PhD dissertation, Huazhong University of Science and Technology, 2011.

18. Fowler JR, Lavasani M, Huard J and Goitz RJ: Biologic strategies to improve nerve regeneration after peripheral nerve repair. J Reconstr Microsurg 31: 243-248, 2015.

19. Hosli E and Hosli L: Receptors for neurotransmitters on astrocytes in the mammalian central nervous system. Prog Neurobiol 40: 477-506, 1993.

20. Hamke M, Herpfer I, Lieb K, Wandelt C and Fiebich BL: Substance $\mathrm{P}$ induces expression of the corticotropin-releasing factor receptor 1 by activation of the neurokinin-1 receptor. Brain Res 1102: 135-144, 2006.

21. Xu G: Investigation on the Interaction Mechanism of Nerve Growth Factor NGF and Neuropeptide SP in Hypertrophic Scar. $\mathrm{PhD}$ dissertation, South China University, 2015.

22. Jia Y: The relationship between exogenous substance $p$ and focal adhesion kinase, protein kinase B and nuclear factor in scar formation. Shanxi Medical University, 2012.

23. Zhao JY, Fu XB and Lei YH: Preparation of small area whole layer skin defect wound model in rats. Infect Inflamm Rep 9: 64, 2008.

24. Shen DX: A Quantitative Method for the Study of Anti-inflammatory Effects of Chinese and Western Medicine. Chin J IntegTradit West Med 3: 49, 1983 (In Chinese).

25. Tang QL: Burn skin regenerative medical technology clinical application specification (Chinese Edition). Chin Trad Chinese Med Press, Beijing, 2013.

26. Ma FS: Accumulative eschar after burn. Clin Case Rep 4: 151-153, 2015.

27. Sakallioglu AE, Basaran O, Karakayali H, Ozdemir BH, Yucel M, Arat Z and Haberal M: Interactions of systemic immune response and local wound healing in different burn depths: An experimental study on rats. J Burn Care Res 27: 357-366, 2006.

This work is licensed under a Creative Commons Attribution-NonCommercial-NoDerivatives 4.0 International (CC BY-NC-ND 4.0) License. 